\title{
Genetic Analysis of a Dictyostelium discoideum Mutant Resistant to Adenosine 3':5'-Cyclic Phosphorothioate, an Inhibitor of Wild-type Development
}

\author{
By E. WALLRAFF,* D. L. WELKER, K. L. WILLIAMS $\dagger$ AND \\ G. GERISCH \\ Max-Planck-Institut für Biochemie, D-8033 Martinsried bei München, \\ Federal Republic of Germany
}

(Received 5 January 1984)

\begin{abstract}
A mutant of Dictyostelium discoideum strain AX2 with altered responses to cyclic AMP has been analysed genetically. The mutant, HG302, aggregates and fruits in the presence of adenosine $3^{\prime}: 5^{\prime}$-cyclic phosphorothioate (cAMPS). This slowly hydrolysed analogue of cyclic AMP inhibits wild-type development by blocking cell differentiation from the growth phase to the aggregation-competent stage. The mutant forms small aggregates in the absence and in the presence of cAMPS, and is further distinguished from the wild type by decreased activities of extracellular phosphodiesterase and phosphodiesterase inhibitor. These phenotypic characteristics were all linked to the cAMPS resistance, which was assigned to linkage group II. Mitotic crossover experiments showed that the cAMPS-resistance mutation, cas A1000, maps proximal to the acr $A$ locus in the neighbourhood of whiA. The data suggest that cas $A 1000$ acts pleiotropically, and that the product of the wild-type allele is required in a part of the signalprocessing pathway common to control of development and regulation of extracellular phosphodiesterase.
\end{abstract}

\section{INTRODUCTION}

Aggregation of single amoeboid cells of Dictyostelium discoideum into a multicellular organism is based on cell cohesion and chemotaxis whereby cyclic AMP (cAMP) acts as the chemoattractant. In the wild-type strain NC4 and its axenic derivative AX2, cAMP also controls cell differentiation from the growth phase to the aggregation competent stage. When applied as periodic pulses, cAMP accelerates development (Darmon et al., 1975; Gerisch et al., 1975). The pulses simulate the natural cAMP signals which are produced by periodic activation of adenylate cyclase (Gerisch \& Wick, 1975; Roos et al., 1977). Pulsatile cAMP signals are necessary for stimulation of development because the response system undergoes rapid adaptation. Continuous application of cAMP interferes with the generation of periodic cAMP signals and delays the acquisition of aggregation competence (Gerisch \& Hess, 1974; Gerisch et al., 1984). Accordingly, a slowly hydrolysed thio-analogue, adenosine $3^{\prime}: 5^{\prime}$-cyclic phosphorothioate (cAMPS), prevents wild-type cells from aggregating in agar plate cultures (Rossier et al., 1978). Addition of cAMP during the preaggregation stage induces extracellular phosphodiesterase and suppresses an inhibitor of this enzyme which, like the phosphodiesterase, is a glycoprotein (Franke \& Kessin, 1981). Neither the induction of extracellular phosphodiesterase nor the suppression of its inhibitor depends on pulsatile cAMP application (Yeh et al., 1978). Similarly, phosphodiesterase activity is raised and the inhibitor is suppressed when cAMPS is added to the cultures (Rossier et al., 1980).

† Present address: School of Biological Sciences, Macquarie University, North Ryde, NSW 2113, Australia.

Abbreviations: ePD, extracellular phosphodiesterase; PDI, phosphodiesterase inhibitor. 
In order to select mutants which do not require pulsatile cAMP signals for development, mutagenized spores were plated on cAMPS-agar and colonies showing aggregation and fruiting body formation were picked (Rossier et al., 1980). Mutant aggregates were always smaller in the presence of cAMPS than were normal wild-type aggregates in its absence. This observation indicates that the interference of cAMPS, present in the medium at a uniform concentration, with chemotactic orientation and signal relay reduces aggregate size.

One of the mutants that aggregated in the presence of cAMPS, HG302, was distinguished from the wild-type by the formation of small aggregates both in the presence and absence of cAMPS, which gave rise to tiny fruiting bodies, and by low extracellular phosphodiesterase and inhibitor activities (C. Rossier, F. Eckstein, M. Westphal \& G. Gerisch, unpublished). This strain was formerly called cAMPSR 302 (Gerisch et al., 1984). We have analysed this mutant genetically in order to map the mutation responsible for resistance of development to cAMPS, and to test whether small aggregate size and the defects of extracellular phosphodiesterase and inhibitor production can be separated from cAMPS resistance by recombination of chromosomes or mitotic crossing over.

\section{METHODS}

Strains of Dictyostelium discoideum. All strains used were derived from the NC4 wild-type (Table 1) and, if not stated otherwise, were grown at $21 \pm 1{ }^{\circ} \mathrm{C}$ in dim light on SM agar plates together with a streptomycin-sensitive, cobalt-resistant strain of Klebsiella aerogenes (Williams \& Newell, 1976).

Selection of diploids. Diploids were constructed as previously described (Newell et al., 1977), except that selection of the diploid DU2413 was based on complementation of the recessive bsgA5 mutation and the dominance of the cob-354 cobalt-resistance mutation (Williams, 1978; Metz et al., 1983). For this selection, the cobalt-resistant Bacillus subtilis strain BSU2, isolated from strain 36.1 by Dr S. Kayman in our laboratory, was incubated overnight on SM agar containing $250 \mu \mathrm{g}$ cobaltous chloride $\mathrm{ml}^{-1}$ and $10 \mu \mathrm{g}$ dihydrostreptomycin sulphate $\mathrm{ml}^{-1}$, to form a thin lawn prior to inoculation with $D$. discoideum.

Haploidization and genetic analysis of recombinants. Diploids were haploidized on SM agar containing $2 \mu \mathrm{g}$ thiabendazole $\mathrm{ml}^{-1}$ (Welker \& Williams, 1980). Haploids were identified by spore size and also by the expression of recessive mutations. Drug resistance was tested by growth on SM agar containing the appropriate inhibitor drugs (Mosses et al., 1975; Williams \& Newell, 1976), and growth temperature sensitivity on SM agar at 26.8 \pm $0.2{ }^{\circ} \mathrm{C}$. Inability to grow on $B$. subtilis was tested on $\mathrm{SM}$ agar plates prespread with a suspension of $B$. subtilis strain 36.1 in $0.1 \mathrm{ml}$ of a solution of $3 \mathrm{mg}$ dihydrostreptomycin sulphate $\mathrm{ml}^{-1}$ (Newell et al., 1977). Spore colour was determined by inspection of fruiting bodies on SM plates. $\alpha$-Mannosidase- 1 activity was determined as described by Free et al. (1976). Mitotic recombinants of linkage group II were selected on the basis of homozygosity for a recessive mutation at the acr $A$ locus as described by Mosses $e t$ al. (1975).

Assays for cAMPS resistance and for extracellular phosphodiesterase and inhibitor production. Cells were grown at $23 \pm 1{ }^{\circ} \mathrm{C}$ in the light, together with Escherichia coli $\mathrm{B} / 2$ on LN (low nutrient) agar containing $17 \mathrm{~mm}$-Soerensen phosphate buffer pH 6.0 plus $0.1 \%$ glucose, $0.1 \%$ peptone (Bacteriological Peptone, Oxoid L37) and $2 \%$ Bactoagar (Difco; 0140-01). This agar was used with or without $2 \mu \mathrm{M}$-cAMPS, which was added from a $0.2 \mathrm{~mm}$ stock solution after melting and cooling the agar to $60^{\circ} \mathrm{C}$. For testing cAMPS resistance, plates of $9 \mathrm{~cm}$ diameter containing $20 \mathrm{ml}$ agar were uniformly inoculated with bacteria, after which cells of the $D$. discoideum strains to be tested were dispensed in the form of nine well-separated spots per plate. Aggregation and fruiting body formation were examined after $3 \mathrm{~d}$. Extracellular cAMP-phosphodiesterase and its inhibitor were assayed from cultures grown in plates of $55 \mathrm{~mm}$ diameter, containing $7 \mathrm{ml} \mathrm{LN}$ agar with or without $2 \mu \mathrm{M}$-cAMPS. The plates were uniformly inoculated with bacteria and with one spot, in the centre, of $D$. discoideum spores. After 3 to $5 \mathrm{~d}$, when the plaques had reached a diameter of $30 \mathrm{~mm}$ or more, the surface of the agar was cleaned with a glass rod, the area beneath the plaque cut out and fluid collected from the agar by centrifugation (Gerisch, 1976). Inhibitor was assayed after inactivating the phosphodiesterase for $10 \mathrm{~min}$ at $80^{\circ} \mathrm{C}$ (Riedel et al., 1973). Free phosphodiesterase was assayed without pretreatment, total phosphodiesterase after inactivating the inhibitor with $0.2 \mathrm{mM}-$ dithiothreitol, according to Franke \& Kessin (1981). Since different batches of dithiothreitol inactivated the enzyme to various extents, controls were run with phosphodiesterase from the inhibitor-less mutant aggr 50 (Riedel et al., 1973), and the values were corrected accordingly.

Chemicals. Cycloheximide was obtained from Sigma; methanol and cobaltous chloride were analytical reagent grade from May \& Baker; thiabendazole was a gift from William Bliss of Merck, Sharp and Dohme Research Labs, Ingleburn, NSW, Australia; cAMPS was a gift from Dr F. Eckstein, Göttingen, FRG. 
RESULTS

\section{Assignment of a cAMPS-resistance mutation to linkage group II}

The cAMPS-resistant mutant, HG302, was isolated from strain AX2, which had no selective marker for genetic analysis. Therefore, to select diploids we used a haploid tester strain, HU1628, which carried the unstable dominant cobalt resistance cob-354 (Williams, 1978; Metz et al., 1983) and also markers on all six established linkage groups (Table 1). The resulting diploid (DU2413) was heterozygous with respect to cAMPS resistance, as well as to the markers of the tester strain (Table 1). The cAMPS-resistance phenotype of HG302 was recessive to wildtype, as diploid DU2413 was cAMPS sensitive. A total of 48 independent segregants obtained from DU2413 were checked for the various marker phenotypes. As shown in Table 2, cAMPS resistance segregated independently of markers on all linkage groups except for linkage group II, in which case the test marker (acrA1823) and cAMPS resistance never cosegregated. Thus the cAMPS-resistance mutation, cas A1000, mapped to linkage group II. While the morphology of the segregants varied due to genetic background effects, the test for cAMPS resistance was

Table 1. Genotypes of strains used

The markers used to score the linkage groups were: I, cycloheximide resistance (cycAl): II, methanol resistance (acrA1823 or acrA1000), cAMPS resistance (casA1000), white spore colour (whiAI), and growth temperature sensitivity (tsgD12); III, inability to grow on $B$. subtilis (bsgA5); IV, white spore colour (whiC351), VI, absence of $\alpha$-mannosidase-1 (manA2); VII, growth temperature sensitivity $(t s g K 21)$. The mutations previously designated $t s g K 2 I$ and couA351 are almost certainly pleiotropic phenotypes of a single mutation (Welker \& Williams, 1982). Markers at the following loci were not scored in this work: axe $A, a x e B$ and $a x e C$ (ability to grow in axenic medium); frt $B$ (distribution of fruiting bodies in concentric rings); oaaA (ability to develop in the presence of $\omega$-aminocarboxylic acids).

For genotypes of additional segregants of DU2413 see Table 5.

\begin{tabular}{|c|c|c|c|c|c|c|c|c|}
\hline \multirow[b]{2}{*}{ Strain } & \multirow[b]{2}{*}{ Parent } & \multicolumn{6}{|c|}{ Linkage group } & \multirow[b]{2}{*}{ Source* } \\
\hline & & I & II & III & IV & VI & VII & \\
\hline HG302 & $\mathrm{AX} 2$ & + & $\begin{array}{l}\operatorname{cas} A 1000 \\
\text { axeA2 } \\
\text { axe } C 2 \\
\text { oaaA2 }\end{array}$ & $a x e B 2$ & + & + & + & 1 \\
\hline HU1628 & HU1493 & $\operatorname{cycAl}$ & $\begin{array}{l}\operatorname{acr} A 1823 \\
\text { axeAI? } \\
\text { axeCI? } \\
\text { oaaAl? }\end{array}$ & bsg $A 5$ & whiC351 & $\operatorname{man} A 2$ & $\begin{array}{l}\text { tsg } K 21 \\
\operatorname{cou} A 351 \\
\text { frt } B 353\end{array}$ & 2 \\
\hline HU1744 & DU2413 & + & $\begin{array}{l}\text { casA } 1000 \\
\text { axeA2 } \\
\text { axeC2 } \\
\text { oaaA2 }\end{array}$ & $b s g A 5$ & + & + & + & 3 \\
\hline HU1834 & HU1744 & & $\begin{array}{l}\operatorname{casA} 1000 \\
\operatorname{axeA2} \\
\operatorname{axeC2} \\
\text { oaaA2 } \\
\operatorname{acr} A 1000\end{array}$ & $b s g A 5$ & + & + & + & 3 \\
\hline TS 12 & NC4 & $c y c A I$ & $\begin{array}{l}\text { whiAI } \\
\text { tsgD12 }\end{array}$ & + & + & + & + & 4 \\
\hline DU2413 & \multicolumn{8}{|c|}{ diploid constructed by fusion of HG302 and HU1628 } \\
\hline $\left.\begin{array}{l}\mathrm{DG} 1 \\
\mathrm{DG} 2\end{array}\right\}$ & \multicolumn{8}{|c|}{ diploids constructed by fusion of HU1834 and TS12 } \\
\hline
\end{tabular}

- Sources: (1) A haploid cAMPS-resistant mutant of AX2 isolated by C. Rossier (unpublished) after NTG ( $N$ methyl- $N$-nitro- $N$-nitrosoguanidine) mutagenesis (Yanagisawa et al., 1967) to about $1 \%$ survival; (2) Welker \& Williams (1984); (3) this work; (4) Katz \& Sussman (1972). 
Table 2. Linkage analysis of the cas 11000 mutation in independently derived haploid segregants of DU2413

$\begin{array}{clcccc}\begin{array}{c}\text { Linkage } \\ \text { group* }\end{array} & \begin{array}{l}\text { Haploid } \\ \text { parents }\end{array} & \text { Genotype } & \text { casAlO00 } & + & \text { Total } \\ \text { I } & \text { HG302 } & + & 4 & 19 & 23 \\ & \text { HU1628 } & \text { cycAI } & 11 & 14 & 25 \\ \text { II } & \text { HG302 } & + & 15 & 0 & 15 \\ & \text { HU1628 } & \text { acr AI823 } & 0 & 33 & 33 \\ \text { III } & \text { HG302 } & + & 4 & 13 & 17 \\ & \text { HU1628 } & \text { bsgA5 } & 11 & 20 & 31 \\ \text { IV } & \text { HG302 } & + & 9 & 15 & 24 \\ & \text { HU1628 } & \text { whiC351 } & 6 & 18 & 24 \\ \text { VI } & \text { HG302 } & + & 9 & 14 & 23 \\ & \text { HU1628 } & \text { manA2 } & 6 & 19 & 25 \\ \text { VII } & \text { HG302 } & + & 2 & 10 & 12 \\ & \text { HU1628 } & \text { tsgK21 } & 13 & 23 & 36\end{array}$

* Linkage group V remains to be established and was not marked in this work. No evidence for the presence of translocations was found with these segregants; segregation of particular linkage groups was not correlated with the segregation of any others.

always unambiguous. To illustrate this, two pairs of segregants with varying linkage groups I, IV, VI and VII are shown in Fig. 1, along with the parent strains.

\section{Ordering the cas A locus on linkage group II}

To order the cas $A$ locus on linkage group II, mitotic crossing-over studies were performed. To simplify the mitotic mapping, it was necessary to combine a methanol-resistance $(a \mathrm{cr} A)$ mutation, localized on linkage group II, in cis with cas A1000. This was done by selecting a spontaneous acrA mutant of the cAMPS-resistant strain HU1744, a haploid segregant of DU2413. The methanol-resistant strain obtained, HU1834, was fused with strain TS12, which has two ordered markers on linkage group II (Table 1), and two diploids (DG1 and DG2) were obtained. Genotypes and phenotypes on linkage group II of these strains are shown in Table 3 (A). Independent mitotic crossover diploids (Mosses et al., 1975) were obtained by plating on methanol agar $10^{4}$ to $10^{5}$ amoebae of DG1 or DG2 per plate and checking the ploidy of the colonies obtained. As well as over 400 haploids, 14 independently derived diploid recombinants were obtained out of about $7 \times 10^{5}$ cells plated. Five of them were cAMPS sensitive (DG4, DG7, DG11, DG12 and DG18) and nine were cAMPS resistant (DG3, DG5, DG6, DG9, DG10, DG15, DG16, DG17 and DG19). For each of the two diploid phenotypes which were obtained, two genetic maps are possible (B, C and D, E respectively, Table 3 ). In an attempt to determine which map was correct, segregant haploids obtained from the 14 diploid strains were tested for cAMPS resistance (casA), spore colour (whiA) and growth inability at restrictive temperature (tsgD).

In segregants from the cAMPS-sensitive diploids, where cas $A$ must be proximal to the crossover site (B, C, Table 3), two classes of haploid phenotypes were found: (1) yellow, cAMPS-resistant, temperature-resistant; (2) white, cAMPS-sensitive, temperature-resistant. The presence of white-spored, temperature-resistant segregants excludes possibility $B$ and confirms that these diploids arose by mitotic crossing over. This means that the chromosomes must have recombined in interval II, i.e. somewhere between whi $A$ and $a c r A$, and that cas $A$ must be proximal to acrA.

From the cAMPS-resistant diploids (D, E, Table 3), only one segregant phenotype was obtained: yellow, cAMPS-resistant, temperature-resistant. This result is compatible with possibility $\mathrm{D}$ (or non-disjunction) but not with $\mathrm{E}$. This means that the chromosomes recombined in interval $\mathrm{I}$, between the centromere and the whiA locus.

Our experiments establish that the cas $A$ locus is proximal to the $\operatorname{acr} A$ locus but do not allow a 

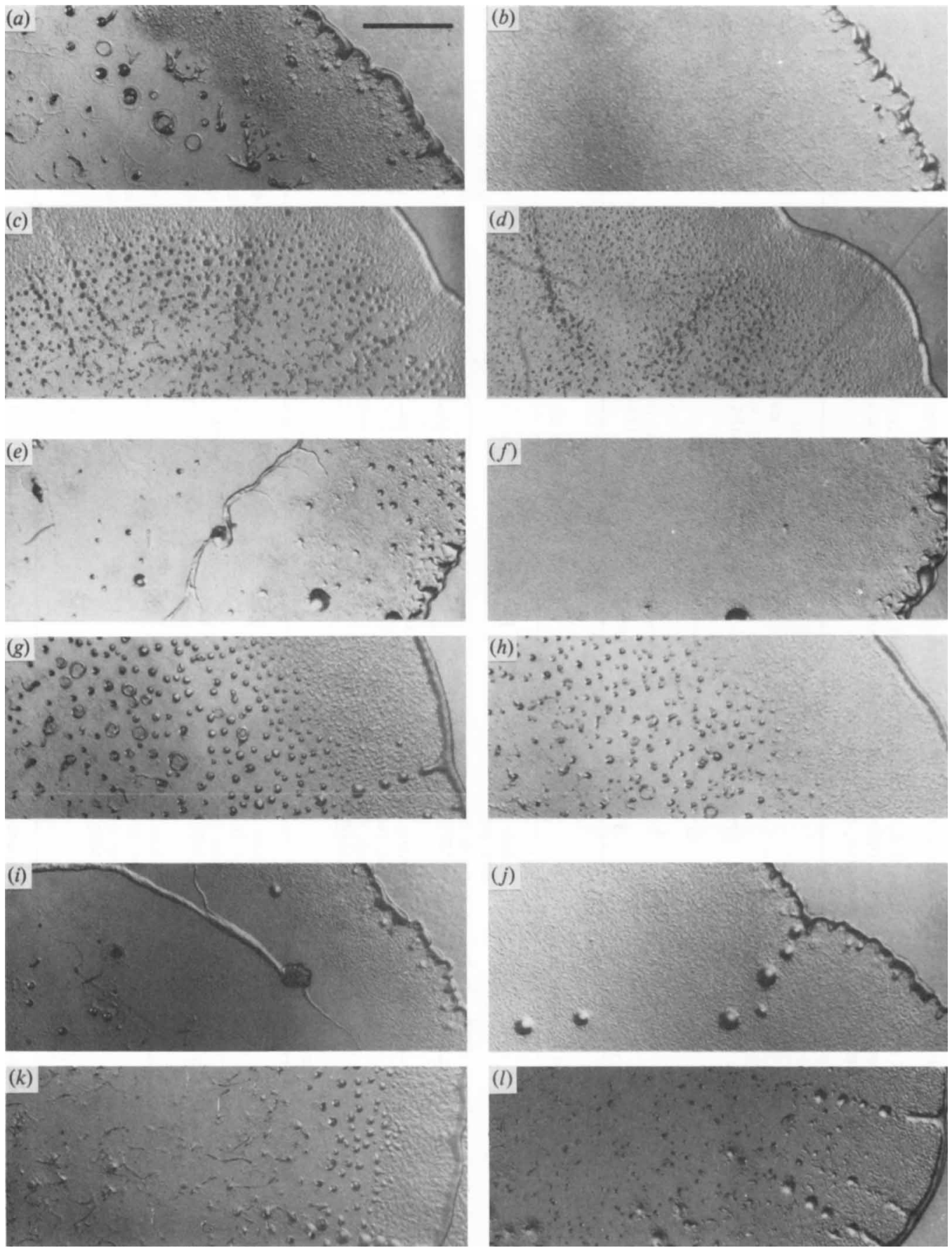

Fig. 1. Cell culture on LN agar plates without cAMPS (left) and with $2 \mu \mathrm{M}$-cAMPS (right). $(a, b)$ cAMPS-sensitive parent strain HU1628; $(c, d)$ cAMPS-resistant parent strain HG302; $(e, f)$ segregant HU1754 (sensitive); $(g, h)$ HU1747 (resistant); $(i, j)$ HU1752 (sensitive); $(k, l)$ HU1749 (resistant). Within each pair of segregants (HU1754/HU1747 and HU1752/HU1749) genotypes were identical on all linkage groups except linkage group II (Table 5). Photographs were taken after $3 \mathrm{~d}$ culture. Bar marker, $2 \mathrm{~mm}$. 


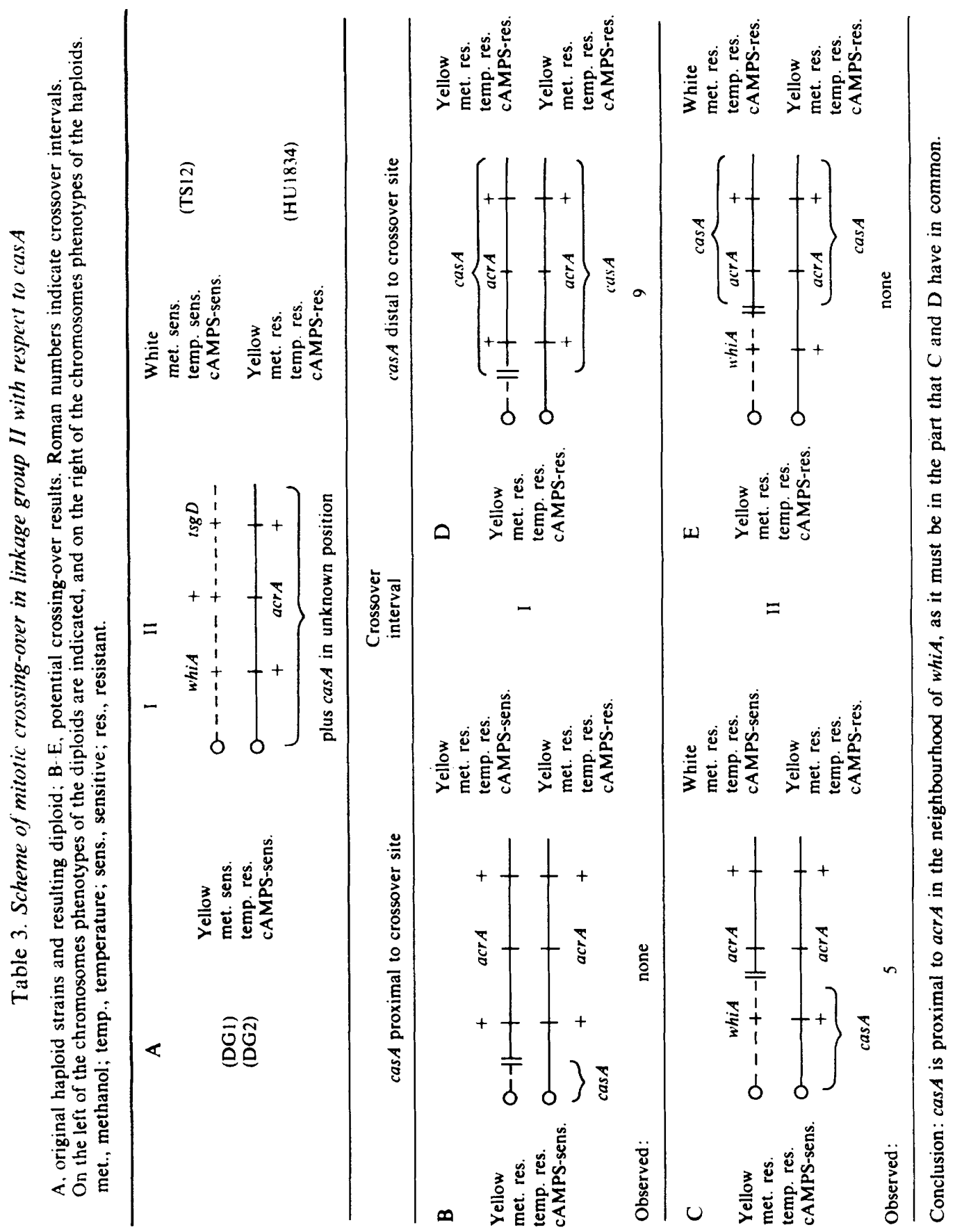


Table 4. Extracellular phosphodiesterase $(e P D)$ and its inhibitor $(P D I)$ produced by the parent strains

Units according to Riedel et al. (1973).

$\begin{array}{lccccc} & \overbrace{\text { ePD }} & \text { PDI } & \overbrace{\text { ePD }}^{\text {LN agar }} & \text { PDI } \\ \text { AX2 (cAMPS sensitive) } & 2.9 & 18 & 33.3 & 7.5 \\ \text { HU1628 (cAMPS sensitive) } & 7.8 & 6.7 & 33.2 & 0.7 \\ \text { HG302 (cAMPS resistant) } & 6.6 & 1.4 & 3.8 & 2.5\end{array}$

Table 5. Genotypes of segregants of DU2413 used for the ePD and PDI assays shown in Figs 2(a) and 3(a)

In the first two columns strains are classified according to the genotype on linkage group II, with those identical on the other linkage groups paired. All linkage group markers were derived from the cAMPSsensitive HU1628 parent of the DU2413 diploid, except cas A1000 which comes from the HG302 mutant (Table 1).

\begin{tabular}{|c|c|c|c|c|c|c|}
\hline \multicolumn{2}{|c|}{ Haploid segregants } & \multicolumn{5}{|c|}{ Linkage group } \\
\hline acr $A 1823$ & casAl000 & I & III & IV & VI & VII \\
\hline HU1751 & HU1743 & cycAl & + & + & $\operatorname{man} A 2$ & $\operatorname{tsg} K 21$ \\
\hline HU1754 & HU1747 & cycAI & bsgA5 & + & $\operatorname{man} A 2$ & $t s g K 21$ \\
\hline HU1746 & HU1744 & + & bsgA5 & + & + & + \\
\hline HU1752 & HU1749 & + & bsgAS & whiC351 & + & + \\
\hline HU1805 & HU1745 & + & bsg $A 5$ & whic 351 & $\operatorname{man} A 2$ & $t \operatorname{sg} K 21$ \\
\hline HU1806 & HU1748 & $c y c A I$ & + & whiC351 & + & $\operatorname{tsg} K 21$ \\
\hline HU1807 & HU1750 & $c y c A I$ & bsgA5 & whiC 351 & + & $t s g K 21$ \\
\hline - & HG8 & cycAl & bsg A5 & + & $\operatorname{man} A 2$ & $t \operatorname{sg} K 21$ \\
\hline - & HG9 & cycAI & bsg A5 & + & $\operatorname{man} A 2$ & $t s g K 21$ \\
\hline - & HG11 & $c y c A I$ & bsgA5 & + & + & $t s g K 21$ \\
\hline - & HG17 & cycAl & bsgA5 & whiC 351 & + & $t s g K 21$ \\
\hline
\end{tabular}

decision about the position of the cas $A$ locus in relation to the whiA locus. However, the two loci are closely linked, as no crossovers between cas $A$ and whiA were observed in any of the 14 crossover diploids examined.

\section{Linkage between $C A M P S$ resistance and decreased production of cAMP phosphodiesterase}

Phenotypically, the mutant HG302 is characterized not only by its resistance to cAMPS but also by a greatly reduced production of extracellular cAMP-phosphodiesterase (ePD). We examined whether or not these qualities map together.

In the original strains, the total ePD activities, measured as the activities of free plus inhibitor-bound phosphodiesterase in the agar, were quite low (Table 4). In the presence of cAMPS, the total ePD activities were greatly increased in cAMPS-sensitive strains AX2 and HU1628, but not in the cAMPS-resistant mutant HG302 (Table 4), in accord with previous observations (Rossier et al., 1980). Similar differences between cAMPS-sensitive and cAMPSresistant strains were found in all haploid segregants of DU2413 that were tested on cAMPScontaining agar. Seven pairs of matched strains were selected (Table 5). Each pair had the same genotype, except for linkage group II, which was different, i.e. marked by cas $A 1000$ in one partner and by acrA1823 in the other. In addition, four further cAMPS-resistant haploid segregants were tested on cAMPS-containing agar. Although the ePD activities measured in different cAMPS-sensitive strains varied considerably, they were consistently, and in most cases quite drastically, higher than in the casA1000-containing strains (Fig. 2a). On agar without cAMPS, all cAMPS-sensitive strains tested showed lower total $\mathrm{ePD}$ activities than on cAMPS agar, indicating that the particularly high levels were not constitutive but induced by cAMPS.

Analogous results were obtained from the haploid segregants derived from the mitotic recombinants (Fig. $2 b$ ). In the haploid segregants derived from four different cAMPS-sensitive 

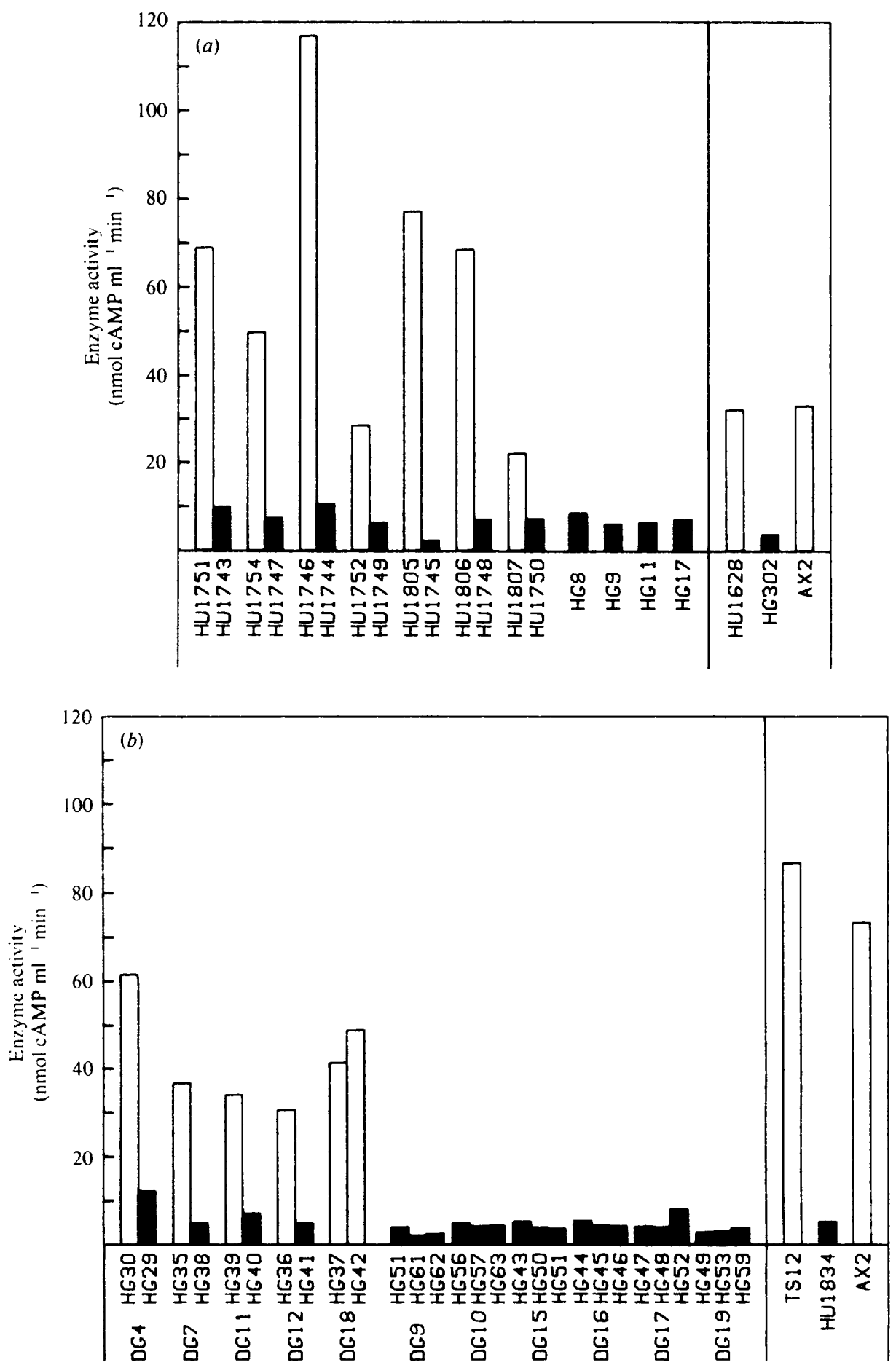

Fig. 2. Total extracellular phosphodiesterase released from cells grown on LN agar containing cAMPS. Open columns, cAMPS-sensitive strains; filled columns, cAMPS-resistant strains. (a) Segregants from DU2413 in comparison to cAMPS-sensitive and resistant original strains. (b) Haploid strains from crossover diploids in comparison to original strains; DG4, DG7, DG11, DG12, DG18 crossover in interval I; DG9, DG10, DG15, DG16, DG17, DG19 - crossover in interval II (Table 3). 

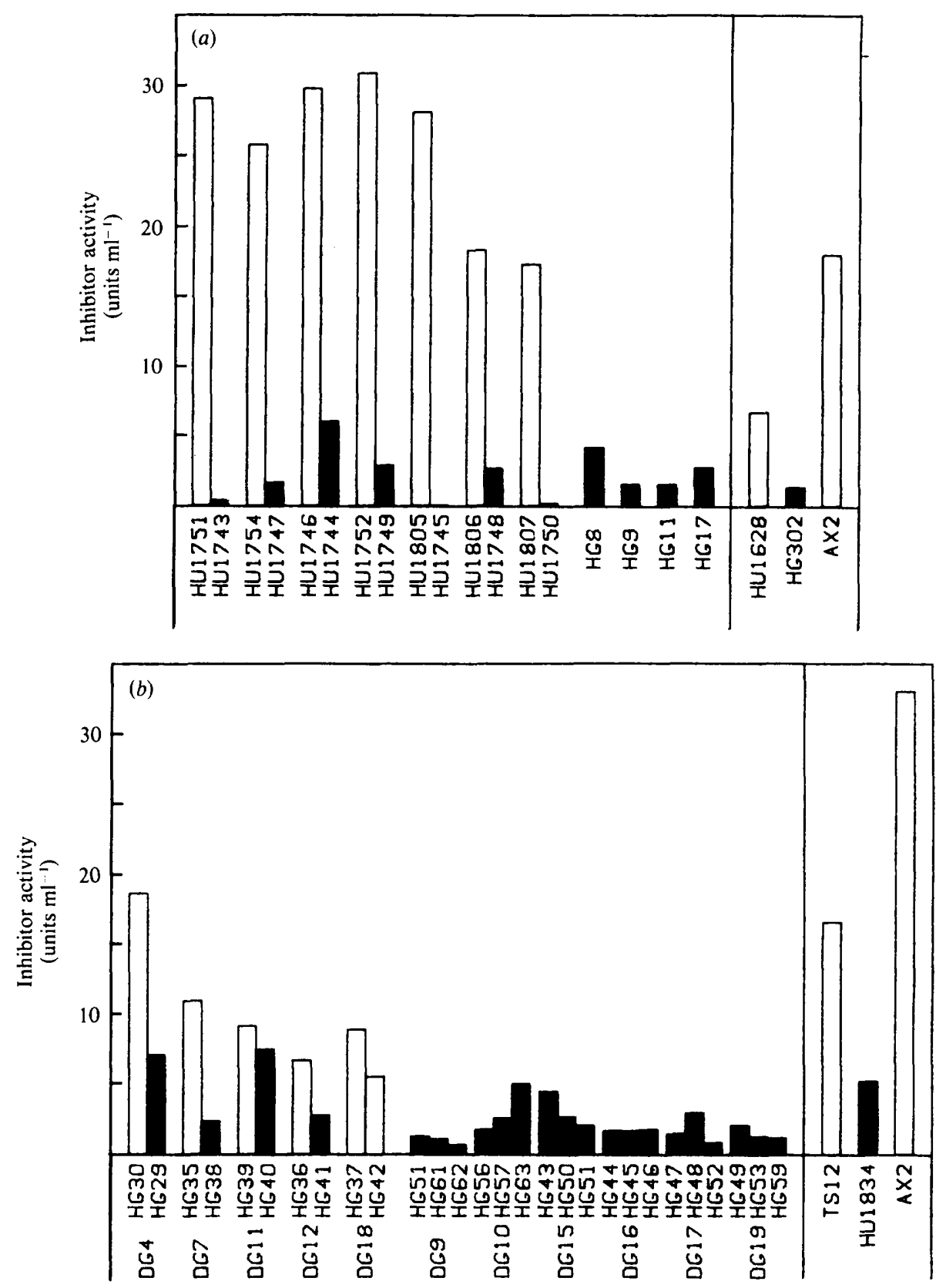

Fig. 3. Phosphodiesterase inhibitor released from cells grown on LN agar without cAMPS. For explanation see legend to Fig. 2, and for definition of units, see Riedel et al. (1973).

( $\operatorname{cas} A^{+} /$cas $A 1000$ ) diploids, ePD activity was decreased in the cas $A 1000$-containing haploids. In the fifth case, both samples investigated were of $\operatorname{cas} A^{+}$type and showed high ePD activities. From each of the nine diploids homozygous for cas $A 1000$, three haploid segregants (all cAMPSresistant) were tested for $\mathrm{CPD}$. Their $\mathrm{ePD}$ activities were as low as those found in other cas $A 1000$ strains, as shown for the segregants of six diploids in Fig. 2(b). Thus, without exception, decreased ePD activity on cAMPS agar was associated with cAMPS resistance. 


\section{Linkage between cAMPS resistance and decreased production of phosphodiesterase inhibitor}

Another constituent of the cAMP regulatory system is the inhibitor of extracellular phosphodiesterase (PDI). In wild-type strains, the inhibitor is suppressed by cAMP or cAMPS (Rossier et al., 1980); in HG302 it is low both in the presence and absence of cAMP or cAMPS (Table 4). With the cAMPS-resistant segregants of DU2413, only low activities of PDI were found in the absence of cAMPS, whereas in the corresponding cAMPS-sensitive cells, the activities were high (Fig. $3 a$ ). Less clear results were obtained from the segregants of the crossover diploids (Fig. 3b). Activities of PDI measured in cAMPS-sensitive segregants were lower than in the cAMPS-sensitive original strains; but on average, cAMPS-sensitive segregants showed higher PDI activities than the cAMPS-resistant ones. Definite conclusions cannot be drawn, however, since the differences between PDI activities of some pairs of cAMPS-resistant and cAMPS-sensitive strains (e.g. HG39, HG40) were only marginal. It remains possible that, while cas $A$ affects inhibitor production, a further mutation is also involved.

\section{Linkage between $\mathrm{CAMPS}$ resistance and small aggregate size}

HG302 cells are able to aggregate yet do not form streams, and their aggregates and fruiting bodies are smaller than in AX2 or HU1628. If cAMPS resistance and small aggregate size are unrelated, segregants might be observed which are cAMPS-resistant but nevertheless form, in the absence of cAMPS, normal-sized aggregates. No segregants investigated behaved in that way. Instead, cAMPS resistance was always associated with the small aggregate size shown in Fig. 1

\section{DISCUSSION}

Genetic analysis of the mutant HG302 has revealed close coupling between the features that distinguish it from the parent strain AX2. These characteristics are the ability to aggregate and fruit in the presence of cAMPS, the formation of small aggregates and accordingly small fruiting bodies, and the suppression of extracellular phosphodiesterase and inhibitor production.

The genetic defect(s) responsible for the phenotypic traits has (have) been mapped to linkage group II, using parasexual genetic analysis. The localization of the pleiotropic phenotypes to one region of linkage group II using mitotic crossing over was, with the exception of inhibitor defects, unequivocal. Thus it is likely that the phenotypic characteristics of the mutant are pleiotropic manifestations of a single gene mutation. Alternatively, they may be caused by two or more mutations in a cluster of genes controlling cAMP-mediated regulation of development and of extracellular phosphodiesterase activities. Common control of development and of extracellular phosphodiesterase is also suggested by a number of mutants obtained from HG302, in which the phosphodiesterase activity was recovered and, simultaneously, development was blocked before aggregation commenced (G. Gerisch \& U. Weinhart, unpublished).

In the cAMPS-sensitive AX2 wild-type strain, extracellular phosphodiesterase regulation is not connected to development in a straightforward way. Periodic pulses of cAMP stimulate and a continuous flux of cAMP inhibits development, although under both conditions the extracellular phosphodiesterase is induced and the inhibitor suppressed. Thus, induction of phosphodiesterase is either linked to stimulation or to inhibition of development, depending on the temporal pattern of cAMP application. It has been concluded that an adaptation step is implemented in the signal-processing pathway connecting enhancement of development to the activation of cell-surface cAMP receptors, and that adaptation does not shut off the signalprocessing pathway linking phosphodiesterase regulation to the receptors (Bernstein et al., 1981). If HG302 contains a single gene mutation, cas A 1000, the gene product affected appears to be a constituent of the proximal part of the signal-processing pathway common to the control of development and of extracellular phosphodiesterase.

HG302 is an unusual cAMPS-resistant mutant, as thirty other cAMPS-resistant mutants not yet analysed genetically aggregate almost normally. They make small aggregates only in the presence of cAMPS (C. Rossier, F. Eckstein, M. Westphal \& G. Gerisch, unpublished). Phosphodiesterase and inhibitor regulation has been examined in three of these mutants and 
shown to be essentially normal. Therefore, in most cAMPS-resistant mutants a region of signal processing specific to the control of development appears to be affected.

It is easy to understand that in the presence of cAMPS the aggregate size is limited, because it would be difficult for the cells to detect, against a uniform concentration of cAMPS, gradients of cAMP for chemotactic orientation. Signal relay, i.e. the propagation of cAMP waves, would likewise be disturbed. With high concentrations of cAMPS in the agar, aggregation occurs largely as a consequence of unorientated movement and adhesiveness between randomly colliding cells (E. Wallraff \& G. Gerisch, unpublished). Less easily explained is the small aggregate size exhibited by HG302 in the absence of cAMPS. It indicates that in HG302 development is not only uncoupled from periodic cAMP signals, as in other cAMPS-resistant mutants, but that the cAMP signal system itself is defective. Whether the defect is in chemotaxis or signal relay remains to be identified.

This work was supported by a grant from the Deutsche Forschungsgemeinschaft. We thank Georg Monok for phosphodiesterase and inhibitor assays.

\section{REFERENCES}

BERNSTEIN, R. L., RosSier, C., VAN DRIEL, R., BRUNNER, M. \& GERISCH, G. (1981). Folate deaminase and cyclic AMP phosphodiesterase in Dictyostelium discoideum: their regulation by extracellular cyclic AMP and folic acid. Cell Differentiation 10, 79-86.

Darmon, M., Brachet, P. \& Pereira da Silva, L. H. (1975). Chemotactic signals induce cell differentiation in Dictyostelium discoideum. Proceedings of the National Academy of Sciences of the United States of America 72, 3163-3166.

Franke, J. \& Kessin, R. H. (1981). The cyclic nucleotide phosphodiesterase inhibitory protein of Dictyostelium discoideum. Journal of Biological Chemistry 256, 7628-7637.

Free, S. J., SCHIMKE, R. T. \& LOOMIS, W. F. (1976). The structural gene for $\alpha$-mannosidase- 1 in Dictyostelium discoideum. Genetics 84, 159-174.

GERISCH, G. (1976). Extracellular cyclic-AMP phosphodiesterase regulation in agar plate cultures of Dictyostelium discoideum. Cell Differentiation 5, 2125.

Gerisch, G. \& Hess, B. (1974). Cyclic-AMP-controlled oscillations in suspended Dictyostelium cells: their relation to morphogenetic cell interactions. Proceedings of the National Academy of Sciences of the United States of America 71, 2118-2122.

GERISCH, G. \& WICK, U. (1975). Intracellular oscillations and release of cyclic AMP from Dictyostelium cells. Biochemical and Biophysical Research Communications 65, 364-370.

Gerisch, G., Fromm, H., Huesgen, A. \& Wick, U. (1975). Control of cell-contact sites by cyclic AMP pulses in differentiating Dictyostelium cells. Nature, London 255, 547-549.

Gerisch, G., Tsiomenko, A., Stadler, J., Claviez, M., HÜlSER, D. \& Rossier, C. (1984). Transduction of chemical signals in Dictyostelium cells. In Information and Energy Transduction in Biological Membranes. Edited by E. Helmreich. New York: Alan R. Liss (in the Press).

KatZ, E. R. \& Sussman, M. (1972). Parasexual recombination in Dictyostelium discoideum: selection of stable diploid heterozygotes and stable haploid segregants. Proceedings of the National Academy of Sciences of the United States of America 69, 495-498.
Metz, B. A., WARD, T. E., Welker, D. L. \& Williams, K. L. (1983). Identification of an endogenous plasmid in Dictyostelium discoideum. EMBO Journal 2, 515-519.

Mosses, D., Williams, K. L. \& Newell, P. C. (1975). The use of mitotic crossing-over for genetic analysis in Dictyostelium discoideum: mapping of linkage group II. Journal of General Microbiology 90, 247259.

Newell, P. C., Henderson, R. F., Mosses, D. \& RATNER, D. I. (1977). Sensitivity to Bacillus subtilis: a novel system for selection of heterozygous diploids of Dictyostelium discoideum. Journal of General Microbiology 100, 207-211.

Riedel, V., Gerisch, G., MÜller, E. \& Beug, H. J. (1973). Defective cyclic adenosine-3' $: 5^{\prime}$-phosphate phosphodiesterase regulation in morphogenetic mutants of Dictyostelium discoideum. Journal of Molecular Biology 74, 573-585.

Roos, W., Scheidegger, C. \& Gerisch, G. (1977). Adenylate cyclase activity oscillations as signals for cell aggregation in Dictyostelium discoideum. Nature, London 266, 259-261.

Rossier, C., Gerisch, G., Malchow, D. \& Eckstein, F. (1978). Action of a slowly hydrolysable cyclic AMP analogue on developing cells of Dictyostelium discoideum. Journal of Cell Science 35, 32.1338.

Rossier, C., Eitle, E., van Driel, R. \& Gerisch, G. (1980). Biochemical regulation of cell development and aggregation in Dictyostelium discoideum. Symposia of the Society for General Microbiology 30, 405424.

Welker, D. L. \& Williams, K. L. (1980). Mitotic arrest and chromosome doubling using thiabendazole, cambendazole, nocodazole and benlate in the slime mould Dictyostelium discoideum. Journal of General Microbiology 116, 397-407.

Welker, D. L. \& Williams, K. L. (1982). Genetic analysis and phenotypic characterization of effects on the cytoskeleton of coumarin-sensitivity mutations in Dictyostelium discoideum. Journal of General Microbiology 128, 1329-1343.

Welker, D. L. \& Williams, K. L. (1984). Translocations in Dictyostelium discoideum. Genetics (in the Press). 
WiLLIAMS, K. L. (1978). Characterization of dominant resistance to cobalt chloride in Dictyostelium discoideum and its use in parasexual genetic analysis. Genetics 90, 37-47.

Williams, K. L. \& Newell, P. C. (1976). A genetic study of aggregation in the cellular slime mould Dictyostelium discoideum using complementation analysis. Genetics 82, 287-307.

Yanagisawa, K., Loomis, W. F. \& Sussman, M.
(1967). Developmental regulation of the enzyme UDP-galactose polysaccharide transferase. Experimental Cell Research 46, 328-334.

Yeh, R. P., Chan, F. K. \& Coukell, M. B. (1978). Independent regulation of the extracellular cyclic AMP phosphodiesterase inhibitor system and membrane differentiation by exogenous cyclic AMP in Dictyostelium discoideum. Developmental Biology 66, 361-374. 